\title{
Gatekeepers and support for research involving people with learning disabilities
}

\author{
Peter Williams \\ Department of Information Studies, University College London, London, UK,
}

Before contributing to the debate around my paper, l'd like to thank everyone who has commented so far. I found colleague' observations thoughtful, supportive and insightful. My responses follow.

First, the Inclusive Research Network (IRN) 'wondered if researchers could approach people with learning disabilities through self-advocacy platforms as they have strategies in place to protect their members and they may be more receptive to research invitations'. Coverdale and Nind also mention 'working directly with people with learning disabilities rather than proxies', recruited in part via self-advocacy groups. Reflecting again on my own experiences, I have indeed found such contacts to be more positive with regard to research possibilities. However, too much reliance on people who may have particular characteristics or be treated in a particular way by virtue of their status as self-advocates, may bias the research. Also, even accessing these groups entails negotiating with 'gatekeepers', with the possible attendant problems mentioned in my paper and, indeed, acknowledged by Coverdale and Nind - that is, the gatekeepers having 'their own ideas about ideal candidates'.

\begin{abstract}
Coverdale and Nind also make the excellent point that some gatekeepers have little power 'in their working lives ... [but] in ... gatekeeping, exercise some power', and feel that my paper lacks consideration to possible power dynamics. I agree it lacks this element. As I outlined, some of the people I approached, rather than finding themselves with 'some power', either referred my request upward or abrogated responsibility by non-engagement. Thus, it could be argued, demonstrated their lack of power. Pallisera makes other points about 'power', suggesting that research that aims to give people more control over their lives will necessarily 'question . . . an institutional culture based on relations of power in which professionals make priority decisions'. As she says, denying access to researchers 'avoid[s] having to make decisions regarding the management of organizational, relational or professional problems linked to [research] results'.
\end{abstract}

It was difficult in the paper to make recommendations in how to deal with non-responses. Coverdale and Nind addressed this (albeit not explicitly) in their observation that outlining 'how research may improve the lives of others' may not be sufficient, as 'researchers have poorly treated people with learning disabilities . . . [and therefore] mistrust of research may not be irrational'. Thus, emphasising good, ethical research practices and the respectful treatment of participants may be as important in facilitating recruitment as describing research outcomes themselves. Pallisera feels that participation in research and its dissemination by professionals could provide 'a training opportunity that contributes to the change of perspectives based on the authority of the professionals'. However, as she acknowledges, some professionals wish to retain power, and thus this 'opportunity' may, conversely, be a disincentive to participate. Another reason for non-participation, not mentioned in the article, is the participant documentation required, which may be daunting to read. Østby notes that 'There are specific requirements for the information and [consent] forms ... [which is] not accessible for some ... participants with intellectual disability'. This applies in the UK too. I was obliged to include information around project funding, its length, and the storage/disposal of data. Of course, all participants should have the right to know these facts. One solution might be to offer to provide information that does not directly affect the participants on request, rather than 'up front'.

Finally, the IRN note that I should have mentioned that "Article 12 of the Convention on the Rights of Persons with Disabilities (CRPD) emphasises that persons with disabilities 'enjoy legal capacity on an equal basis with others in all aspects of life'. Good point!

\section{Disclosure statement}

No potential conflict of interest was reported by the author.

CONTACT Peter Williams peter.williams@ucl.ac.uk http://orcid.org/0000-0002-8628-239X 